ENCYCLOPEDDIE Encyclopédie berbère

BERBERE

10 | 1991

10 | Beni Isguen - Bouzeis

\title{
Bou Nouara (mer de)
}

\section{G. Camps}

\section{OpenEdition}

Journals

Édition électronique

URL : http://journals.openedition.org/encyclopedieberbere/1793

DOI : 10.4000/encyclopedieberbere.1793

ISSN : 2262-7197

\section{Éditeur}

Peeters Publishers

\section{Édition imprimée}

Date de publication : 1 décembre 1991

Pagination : 1570-1577

ISBN : 2-85744-549-0

ISSN : 1015-7344

\section{Référence électronique}

G. Camps, «Bou Nouara (mer de) », Encyclopédie berbère [En ligne], 10 | 1991, document B93, mis en ligne le 01 juin 2013, consulté le 24 septembre 2020. URL : http://journals.openedition.org/ encyclopedieberbere/1793; DOI : https://doi.org/10.4000/encyclopedieberbere.1793

Ce document a été généré automatiquement le 24 septembre 2020

(c) Tous droits réservés 


\section{Bou Nouara (mer de)}

\section{G. Camps}

1 La vaste nécropole mégalithique qui couvre le versant ouest et le sommet du Djebel Mazela près du village de Bou Nouara est l'une des plus célèbres d'Algérie orientale. Le Djebel Mazela est un anticlinal de calcaires aptiens qui dépend du massif de l'Oum Settas, à trente kilomètres au sud-est de Constantine. Le nombre des dolmens de cette nécropole est considérable; tous les auteurs s'accordent pour l'estimer à plusieurs milliers. Cette estimation est confirmée par l'examen d'une photographie aérienne au 5 $000^{\mathrm{e}}$; sur cette photographie nous avons pu repérer 410 monuments circulaires et nous estimons que ce chiffre ne représente pas le dixième des dolmens et autres sépultures existants. Le plus grand nombre de monuments de Bou Nouara ont moins de $4 \mathrm{~m}$ de diamètre ; or, sur la photographie seuls les dolmens ayant plus de $8 \mathrm{~m}$ de diamètre sont repérables, encore faut-il qu'ils possèdent un socle qui se traduise par une tâche claire sur la photographie. En tenant compte des innombrables destructions perpétrées au cours des siècles dans ce site d'accès facile, on peut estimer entre 3000 et 4000 le nombre total des dolmens de cette nécropole qui est la plus importante et la plus étendue de toute l'Afrique du nord. 


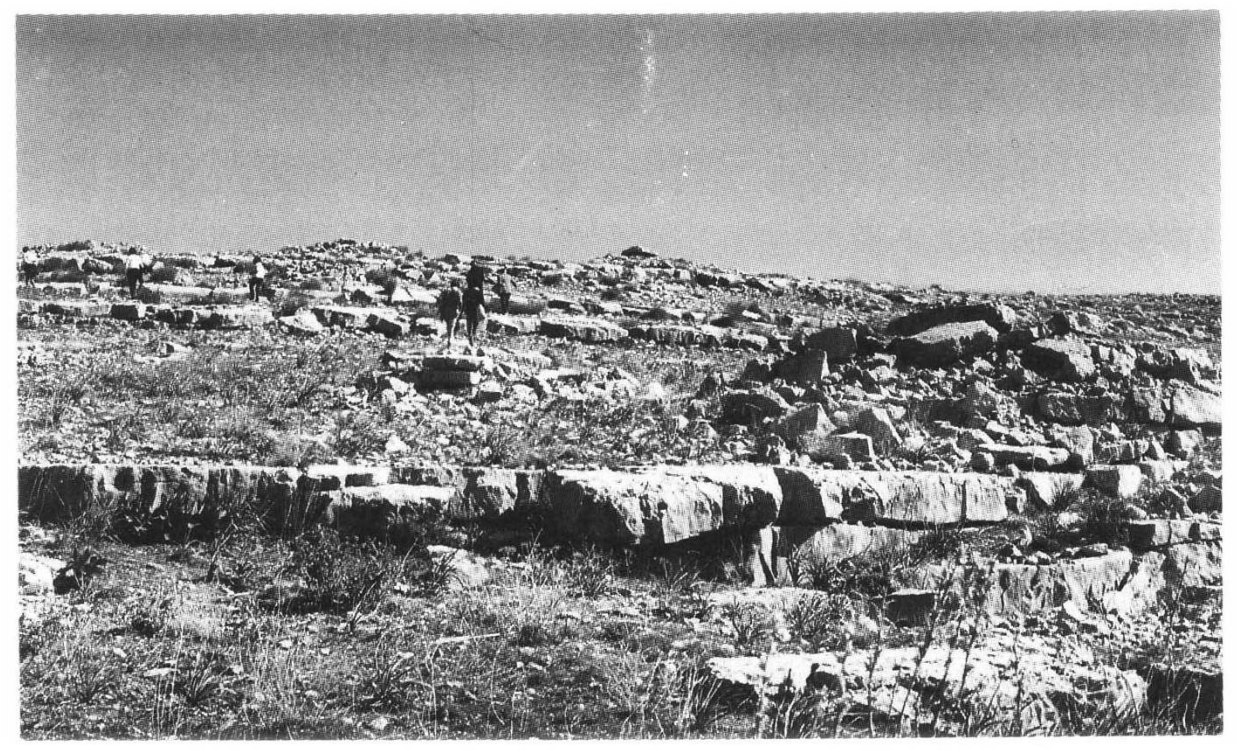

2 Les dolmens de Bou Nouara sont bien visibles de la route et de la voie ferrée de Constantine à Tunis qui empruntent toutes deux la vallée de l'oued Mehiris (qui en aval prend le nom d'oued Berda), aussi la nécropole fut-elle souvent décrite et visitée. La première mention scientifique, celle du général Faidherbe, date de 1868, elle rend compte des fouilles qu'il avait pratiquées dans cinq monuments en 1865. Aucune publication en revanche ne mentionne les fouilles de P. Pallary en 1909 ; c'est grâce à l'étiquette que porte un vase du Musée de Préhistoire du Bardo, à Alger, que l'on sait qu'il fouilla au moins 12 monuments. Quelques années plus tard, en 1913, A. Debruge et L. Joleaud ouvrirent 25 dolmens. Nous-mêmes y avons effectué la fouille de 22 dolmens et 2 bazinas* en 1954, ce qui porte à 42 le nombre de monuments fouillés en un siècle. Ce qui est relativement faible pour une nécropole aussi importante et étendue mais extrêmement pauvre en mobilier funéraire.

3 Les fouilles de 1954 ont apporté une contribution intéressante à l'étude de l'architecture des dolmens et à leur mode de construction. Le calcaire du Djebel Mazela se délite en strates régulières qu'il est facile de débiter en dalles de couverture, en orthostates et en parpaings parallélépipédiques, de ce fait les monuments de ce site paraissent plus soignés que ceux des autres nécropoles comme Beni Messous* ou Roknia*. Indépendamment des facilités offertes par la lithologie locale, il importe cependant de reconnaître le soin apporté à la construction des monuments. Le souci systématique d'élever les dolmens sur un sol en pente répond à une pratique fort simple qui permettrait de faire glisser depuis le haut la dalle de couverture sans avoir à la soulever considérablement au-dessus du sol. Ces dalles étaient facilement détachées des strates supérieures du djebel et ont les faisait glisser, vraisemblablement sur des rouleaux, jusqu'au-dessus des orthostates ou des murets en pierre sèche qui délimitaient, en contrebas, la chambre funéraire. Si la pente facilitait la construction, elle pouvait en revanche se révéler néfaste à la bonne conservation des dolmens, aussi les architectes mégalithiques prirent-ils soin d'étayer le monument dans le sens de la pente en construisant un véritable socle dissymétrique qui rétablissait l'horizontalité. 
Dolmen dégagé de Bou Nouara (Photo G. Camps).

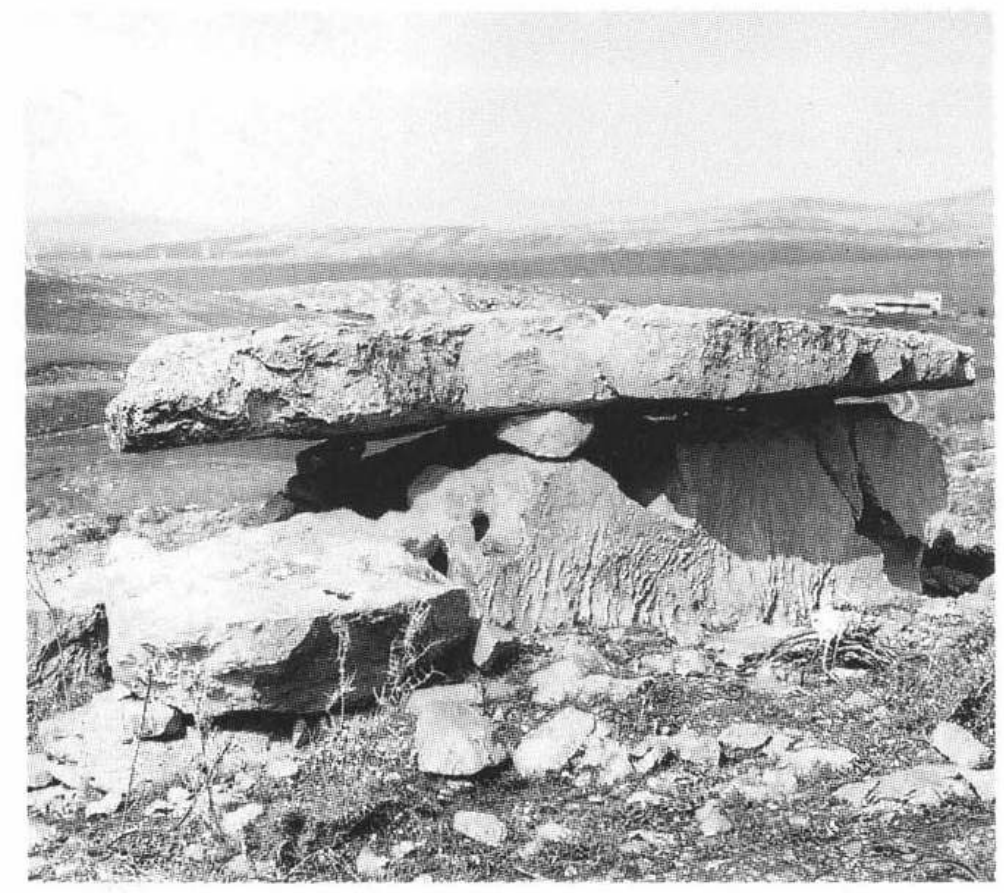

Au premier plan dolmen enfoui, au second plan dolmen engagé dans son socle (Photo G. Camps).

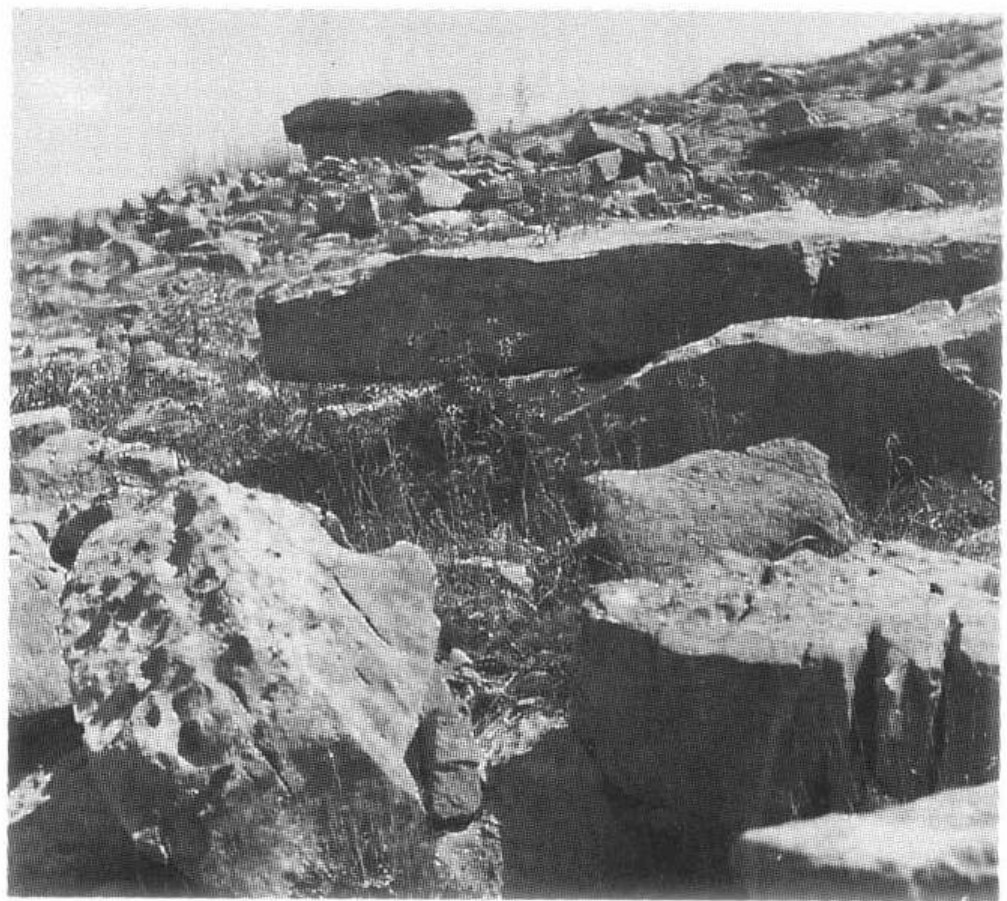

4 Les relations de la chambre funéraire et du socle sont très variables. Nous les avons représentées d'une manière synthétique dans le tableau des différents types de 
dolmens. Indépendamment de l'aspect plus ou moins monumental du socle, le dolmen peut être dégagé, c'est-à-dire qu'il domine largement le socle, engagé, lorsque le haut des orthostates et la dalle de couverture dépassent le niveau du socle, enfoui, lorsque la chambre est entièrement contenue à l'intérieur du socle ou manchon et que la table ne fait qu'affleurer la surface de ce dernier. Dans certains cas le manchon est si important que, pour réduire le volume des matériaux à manipuler, les constructeurs ont aménagé des degrés ou gradins dans l'enceinte du monument. Dans des dolmens de construction particulièrement soignée un escalier a été aménagé pour descendre depuis la surface du socle dans la chambre funéraire.

Il faut reconnaitre que l'architecture des plus grands dolmens, ceux qui, hélas, furent le plus tôt visités et pillés, est bien plus savante que ne le laisserait croire leur apparente rudesse. Les parpaings choisis pour la construction de l'enceinte du socle sont parfois grossièrement taillés en voussoirs pour assurer la régularité de la circonférence. J'ai noté également l'application dans la construction de l'appareil à crochet qui assure l'horizontalité des assises; enfin un système astucieux de demi-cercles excentriques, à l'extérieur de l'enceinte, permet de réduire les travaux de soutènement des monuments. Les mêmes observations ont été faites sur les bazinas qui ne se distinguent architecturalement des dolmens que par l'absence de dalle de couverture. Les monuments sont de dimensions très variables. J'ai pu noter que les grands monuments, dolmens enfouis dans un manchon ou vastes bazinas à degrés, étaient plus nombreux au sommet que sur le versant.

Dolmen enfoui dans son manchon au sommet du Djebel Mazela (Photo M. Gast).

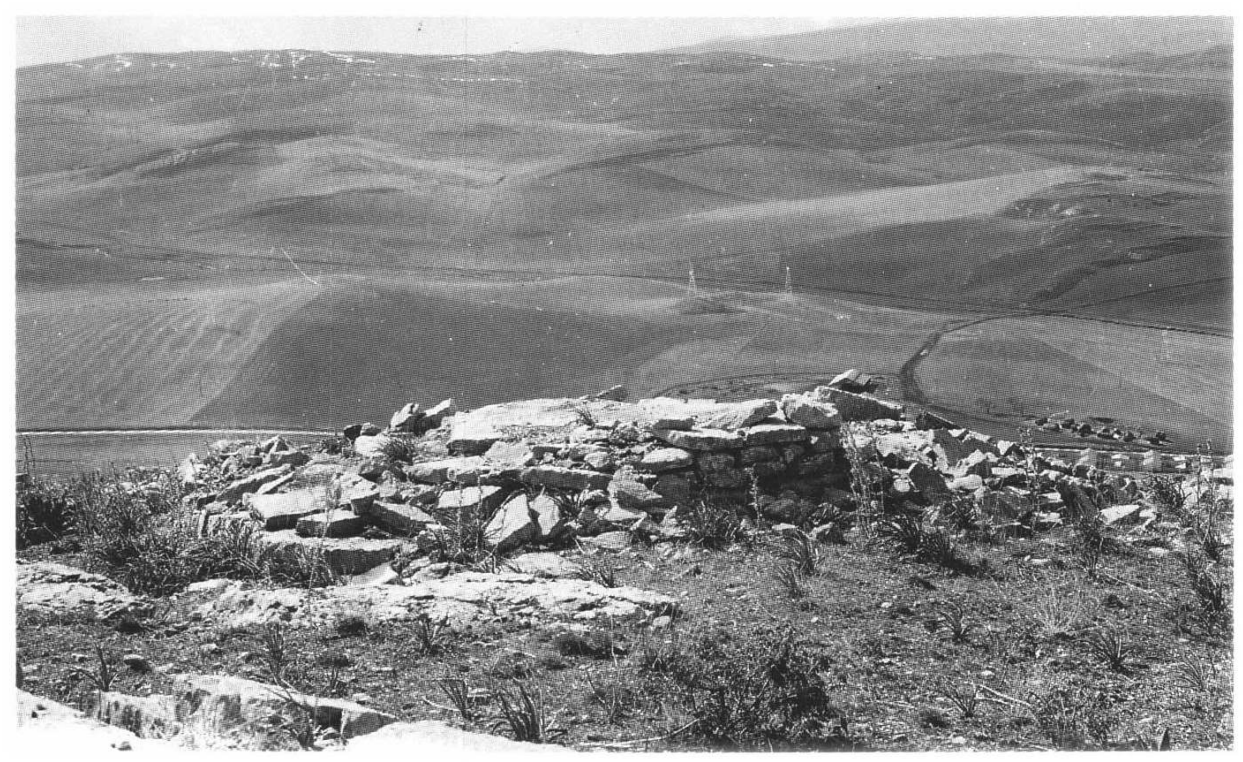

Ces monuments funéraires ne contenaient que fort peu de restes humains et dans une disposition telle qu'il est manifeste que des corps entiers n'avaient pas été ensevelis dans ces chambres. Les ossements, le plus souvent réduits à l'état de faibles esquilles, les dents dispersées sur toute la surface de la sépulture, l'absence totale de connexion font croire à un décharnement préalable. Celui-ci ne fut pas obtenu par l'incinération, ni semble-t-il dans une sépulture provisoire; le mauvais état général des ossements ferait plutôt penser à une exposition sur une aire voisine de la nécropole. Toutefois 
l'une des deux bazinas fouillées en 1954 renfermait, dans le niveau le plus profond, le squelette complet d'un personnage qui avait été enterré accroupi dans l'angle sud de la chambre, un autre corps ou des restes décharnés dans la partie nord et un peu plus tard un troisième individu dont les ossements avaient été déposés au centre, à un niveau plus haut que les précédents. Chacune des ces inhumations avait été accompagnée du dépôt d'une poterie. Au cours d'une seconde période, la sépulture connut un certain bouleversement consécutif à l'introduction d'ossements décharnés dans les parties sud et nord. Au cours de ce remaniement, l'inhumation centrale, située à un niveau supérieur aux deux plus anciennes, fut bouleversée, la jatte d'accompagnement fut brisée ; une partie de cette poterie et certains ossements furent rejetés de la chambre et placés dans l'antichambre. En même temps furent déposées dans cette antichambre deux poteries qui accompagnaient certainement les ossements décharnés introduits dans le monument à ce moment.

Dolmens de Bou Nouara. En haut à gauche, dolmen dégagé sur plate-forme. En bas, dolmen engagé dans un socle à deux assises avec appareil à crochet. A droite, grand dolmen enfoui dans un manchon à degrés.
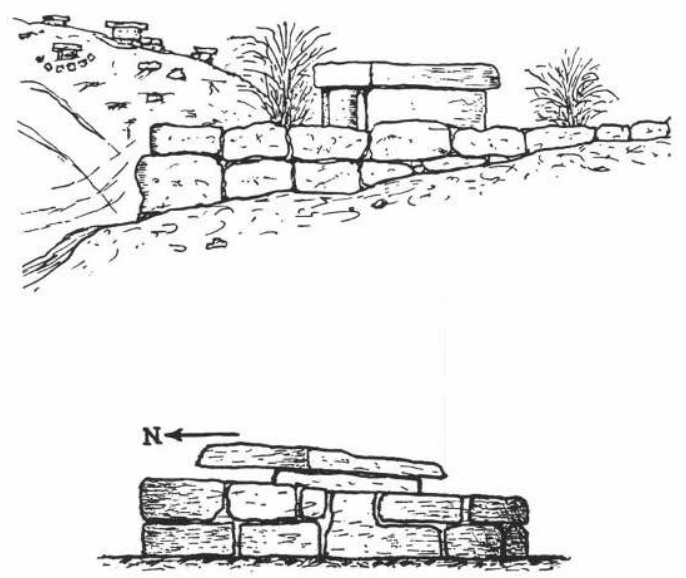

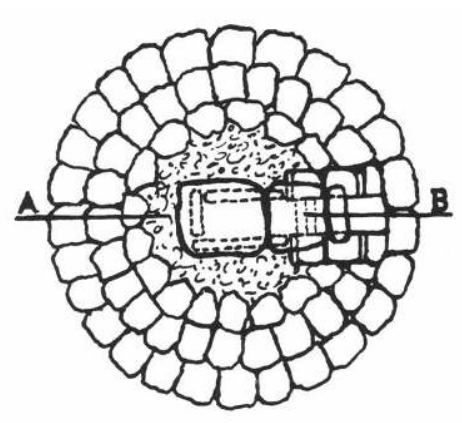

COUPE $A B$

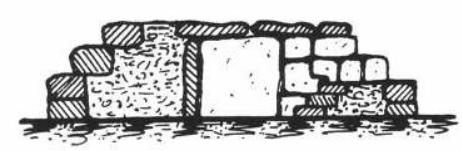


Tableau des différents types de dolmens représentés à Bou Nouara.

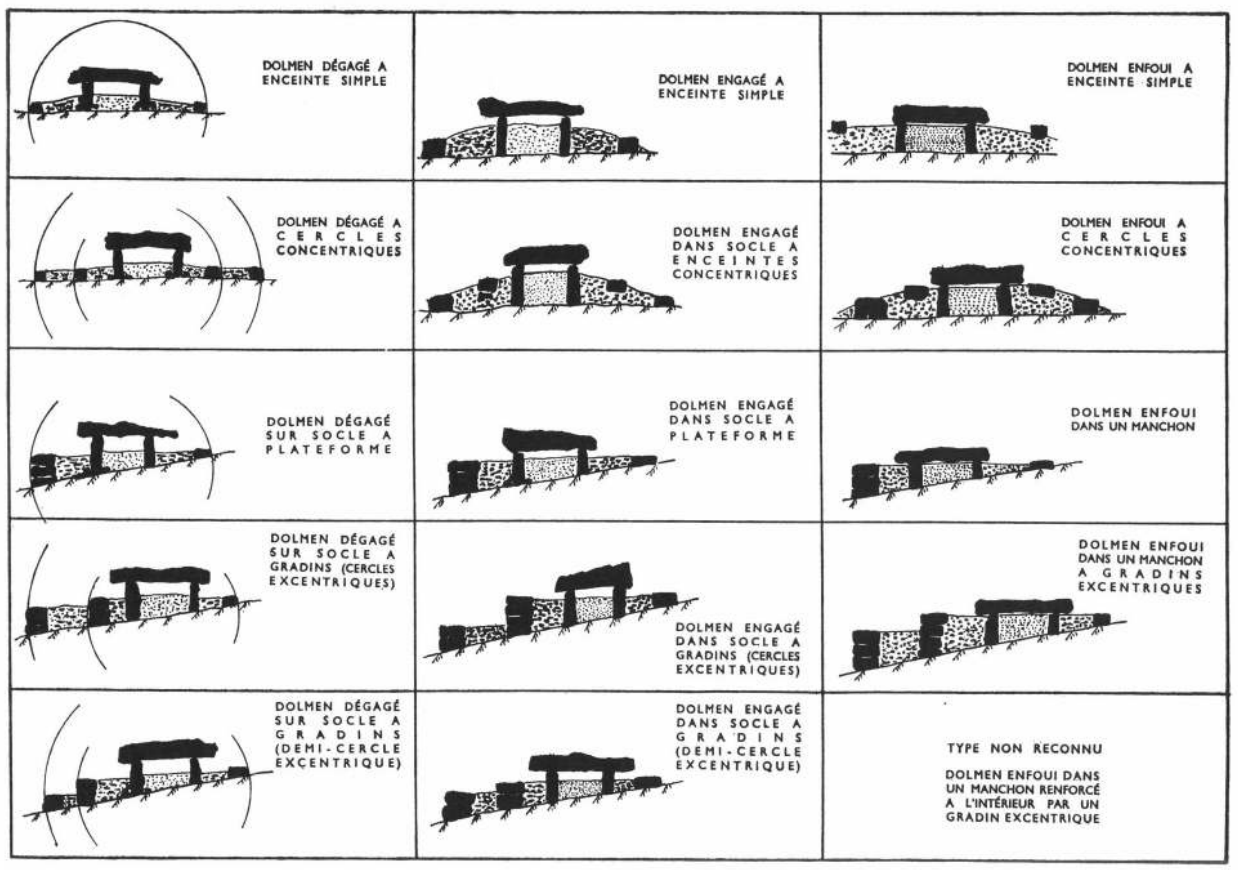

7 Le mobilier funéraire des monuments de Bou Nouara est très pauvre, réduit au minimum, c'est-à-dire un vase ou un fragment de vase par sépulture. Les formes de poterie modelée sont les plus banales et les plus simples du répertoire céramique berbère. Ce sont des bols, des gobelets et des jattes, c'est-à-dire des bols ou des écuelles dont les parois sont carénées. Elles reproduisent, mais en réduction, les formes de la vaisselle domestique les plus usuelles, celles qui servent à contenir la boisson ou les aliments prêts à être consommés. Une pièce exceptionnelle échappe à cette banalité, c'est le seul vase fait au tour découvert dans la nécropole par Debruge et Joleaud. Il s'agit d'une cruche plutôt que d'une oenochoé (bien qu'elle ait le bec légèrement pincé) dont la panse presque sphérique est striée de nombreux sillons parallèles; le décor consiste en deux lignes brisées peintes en brun foncé sur l'épaule. Cette poterie semble dater du IIIe ou du début du IIe siècle av. J.-C. C'est la seule donnée chronologique qu'ait fournie le mobilier funéraire, encore peut-il s'agir d'une introduction tardive. Il est sûr, en revanche, que la fréquentation de cette nécropole dura fort longtemps puisque des ossements de la bazina décrite supra ont été datés par le C 14 de $250 \pm 100$ après J.-C.

Le mobilier métallique est encore plus pauvre que la céramique, on ne signale que deux bracelets en fil de bronze, un anneau de fibule en oméga, une bague et un clou en fer. Les offrandes animales consistent en de rares ossements de moutons, une dent de gazelle, un squelette d'oiseau de la taille d'un passereau.

9 Les monuments de Bou Nouara sont dans l'ensemble orientés nord-sud, conformément à la disposition générale du versant. Mais la fouille des socles nous apporta la preuve que lorsque les constructeurs voulaient observer une orientation rigoureuse, ils ne se laissaient pas guider par les facteurs topographiques; ainsi dans le dolmen IX (fouilles de 1954) le déblaiement du socle jusqu'au rocher permit de découvrir que l'orientation nord-sud avait été donnée à la chambre malgré la présence d'arêtes rocheuses orientées nord-est sud-ouest qui provoquaient des dénivellations sensibles que les 
constructeurs furent contraints de rattraper en glissant des blocs sous une partie des supports est et sud.

Il est une construction, située à mi-pente sur le flanc sud-ouest du Djebel Mazela qui attire l'œil de tout visiteur de Bou Nouara et nourrit l'imagination. Actuellement ce monument cyclopéen est constitué d'un mur de $2 \mathrm{~m}$ de hauteur et long de $8 \mathrm{~m}$, bordé à l'est d'une assise de blocs alignés formant un angle droit avec le mur sur une longueur de $4 \mathrm{~m}$, à l'ouest apparaît la même disposition mais sur $8 \mathrm{~m}$ de longueur. Les blocs de cette construction sont grossièrement équarris et atteignent des dimensions considérables, le plus grand à 1,80 $\mathrm{m}$ de longueur, $1 \mathrm{~m}$ de largeur et $0,50 \mathrm{~m}$ de hauteur. Les fouilles pratiquées à l'intérieur et à l'extérieur de ce singulier monument n'apportèrent aucun enseignement. La destination de ce monument était vraisemblablement cultuelle; on peut imaginer qu'il servit peut-être d'aire d'exposition mais nous n'en avons aucune preuve.

11 Les constructeurs des dolmens et des bazinas de Bou Nouara étaient des paysans, comme le prouve leur vaisselle plate, et éleveurs de moutons qui constituaient l'essentiel des offrandes funéraires animales; ils ne semblent pas avoir connu l'opulence à en juger par la pauvreté du mobilier funéraire. Leurs habitations n'étaient certainement pas sur le Djebel Mazela, dont le nom pourrait évoquer d'anciens sacrifices (de la racine berbère ezlu : égorger), mais dans la vallée, à proximité de leurs champs. Leurs mapalia (gourbis) de terre et de paille ne laissent guère de traces dans des sols cultivés depuis des siècles.

Mobilier des dolmens de Bou Nouara.

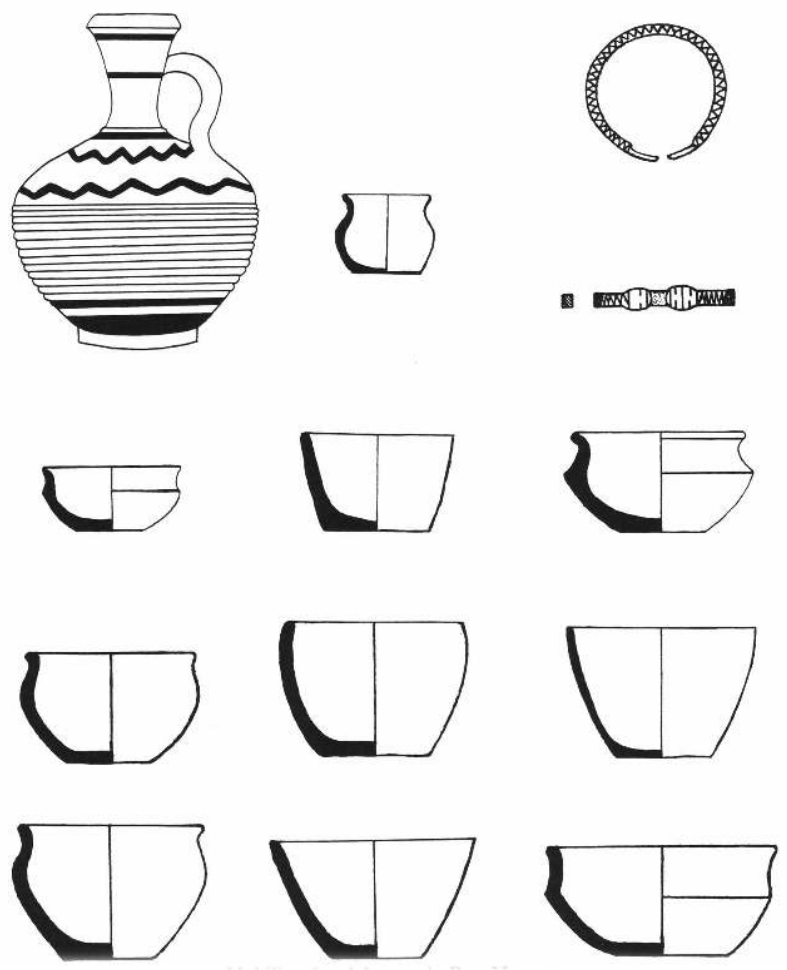

12 L'importance de la nécropole fait penser que ce rassemblement de sépultures était le fait non pas d'un seul groupe résidant dans les parages immédiats mais plutôt de plusieurs clans dispersés appartenant peut-être à une même tribu. Il est très regrettable que le petit nombre de vases conservés ne permette pas de comparer les 
courbes d'absorption, comme cela a été fait dans l'étude du mobilier céramique de Roknia où l'existence de tels groupes a pu être suggérée grâce à ces comparaisons.

\section{BIBLIOGRAPHIE}

Faidherbe Gal, Nécropole mégalithique de Mazela. Bull, de l'acad. d'Hippone, t. V, 1868, p. 63-65.

Id., Les dolmens d'Afrique. Congr. intern. d'Anthrop. et d'Archéol. préhist., $6^{e}$ session, Bruxelles, 1872, p. 406-424.

Féraud L., Monuments dits celtiques dans la Province de Constantine. Rec. des Not. et Mém. de la Soc. archéol. de Constantine, t. VII, 1863, p. 214-234 et t. VIII, 1864, p. 108-132.

Jullien Lt., Excursion à la nécropole mégalithique de Bou Nouara. C.r. du X $X^{e}$ Congr. de l'A.F.A.S., Ager, 1881, p. 1135-1137.

Id., Excursion de M. Henri Martin aux stations mégalithiques des environs de Constantine. Rec. des Not. et Mém. de la Soc. archéol. de Constantine, t. XXII, 1882, p. 214-221.

Gsell S., Monuments antiques de l'Algérie, Paris, 1901, t. 1, p. 23.

Randall Mac Iver D. et Wilkin A., Libyan Notes, Londres, 1901, p. 81-82.

Jacquot L., Quelques dolmens d'Algérie. Bull, de la Soc. préhist. franc., t. VI, 1909, p. 322-324.

Joly A. et Jolaud L., Ruines et vestiges anciens relevés dans la Province de Constantine, Rec. des Not. et Mém. de la Soc. archéol. de Constantine, t. XLIV, 1910, p. 29-34.

Debruge A. et Joleaud L., Contribution à l'étude de la nécropole mégalithique de Bou Nouara, Rec. des Not. et Mém. de la Soc. archéol. de Constantine, t. L, 1916, p. 175-186.

Camps G., Aux origines de la Berbérie. Monuments et rites funéraires protohistoriques, Paris, 1961, p. 125-139 et 487-490.

Camps G. et Camps-Fabrer H., La nécropole mégalithique du Djebel Mazela à Bou Nouara, Paris, 1964.

\section{INDEX}

Mots-clés : Algérie, Protohistoire 